\title{
Bilateral Invasive Lobular Carcinoma Arising within a Fibroadenoma:
} A Rare Case Entity

\author{
Aditi Arora, ${ }^{1, *}$ Ankur Bansal, ${ }^{2}$ Ritika Jaiswal, ${ }^{3}$ and Nuzhat Husain ${ }^{3}$ \\ ${ }^{1}$ MD, PDCC, Department of Pathology, Dr. Ram Manohar Lohia Institute of Medical Sciences, Lucknow, Uttar Pradesh, India \\ ${ }^{2}$ M.Ch PDF, Department of Urology and Kidney Transplant, Sanjay Gandhi Post Graduate Institute of Medical Sciences, Lucknow, Uttar Pradesh, India \\ ${ }^{3}$ MD Department of Pathology, Dr. Ram Manohar Lohia Institute of Medical Sciences, Lucknow, Uttar Pradesh, India \\ "Corresponding author: Dr. Aditi Arora, Senior Resident, PDCC (Onco-Pathology), Department of Pathology, Dr. Ram Manohar Lohia Institute of Medical Sciences, Lucknow, \\ Uttar Pradesh, India. Tel: +91-9559158220, E-mail: draditiarora87@gmail.com
}

Received 2017 January 25; Revised 2017 March 06; Accepted 2017 April 08.

\begin{abstract}
Fibroadenoma is the most common benign tumor of the breast. It may be associated with proliferative epithelial changes, fibrocystic changes, and very rarely, insitu and invasive cancer. This report describes malignant transformation of a bilateral fibroadenoma. The histopathological characteristics and clinical course of a rare case of invasive bilateral lobular carcinoma arising within fibroadenoma in a young female are discussed.
\end{abstract}

Keywords: Bilateral, Invasive, Lobular Carcinoma, Fibroadenoma

\section{Introduction}

Fibroadenoma is a benign breast disorder most commonly affecting females. The two components of fibroadenoma are stroma and epithelium. It may occur as a single lesion (simple or complex) or multiple tumors. It is mostly considered a benign tumor, yet very rarely complex or multiple fibroadenomas may transform to invasive carcinoma. Its epithelial component is at risk of malignant transformation. The risk of this transformation is the same as epithelial elements in the rest of the breast parenchyma. Coexistence of fibroadenoma and carcinoma is a rare entity, and there is no well-documented etiological association between these two conditions. The optimal treatment plan of patients with breast carcinoma within fibroadenoma has not been fully understood. There is very little information regarding this in the current guidelines on breast cancer management (1). It is not known whether these patients should be treated according to the guidelines similar to breast cancer. There are around 160 cases of breast carcinoma within fibroadenoma cases reported in the English literature, and in about 100 cases, malignant transformation of fibroadenoma has been reported (2). This study presents a rare case of malignant transformation of bilateral multiple fibroadenomata to massive sized lobular invasive carcinoma in a 23-year-old female. This case is extremely rare both due to age of the patient at presentation and size of the tumor.

\section{Case Presentation}

A 28-year-old female had presented multiple varying sized lumps (largest measuring $3 \times 3 \mathrm{~cm}$ ) in both breasts 5 years ago. These lumps were diagnosed as simple fibroadenomas on fine needle aspiration cytology (FNAC). She refused to undergo an excisional biopsy. The lumps size had increased rapidly in the last 4 months. On clinical examination, lumps occupied almost her entire breast and measured approximately $15 \times 15 \mathrm{~cm}$ in the right breast and 15 $\times 13 \mathrm{~cm}$ in the left breast with discoloration of overlying skin. Multiple mobile, enlarged bilateral axillary lymph nodes were also palpable (Figure $1 \mathrm{~A}$ ). There was no family history of breast cancer or ovarian cancer. The patient had regular menstrual cycles. Ultrasound and Magnetic Resonance Imaging (MRI) of the breast with angiography revealed highly vascular bilateral multinodular masses involving all quadrants (Figure 1B). Computed Tomography (CT) scan of chest and abdomen did not show any metastasis of lungs or liver. The bone scintigraphy with Tc 99 was normal. A percutaneous trucut biopsy of bilateral lesion was done, which revealed atypical ductal hyperplasia with Carcinoma In Situ (CIS) in her right breast and invasive carcinoma in her left breast. The patient received neo-adjuvant chemotherapy treatment, which consisted of $500 \mathrm{mg} / \mathrm{m}^{2}$ cyclophosphamide (day 1), $50 \mathrm{mg} / \mathrm{m}^{2}$ adriamycin (day 1), and $500 \mathrm{mg} / \mathrm{m}^{2}$ 5-flurouracil (day 1) [CAF regimen]. Three cycles were given at an interval of 3 weeks. The patient responded to chemotherapy and bilateral tumor size decreased. Subsequently, bilateral modified radical mastectomy with axillary lymph node dissection was 
performed. Unfortunately, both the breasts revealed a tumor involving all 4 quadrants of the breast. The tumor was firm to hard, tan white in color, and multinodular with areas of haemorrhage and necrosis (Figure 2). On microscopic examination, there was proliferation of small cells, which were singly scattered and at places arranged in Indian file pattern through a fibrous connective tissue. Occasionally, these cells were arranged in concentric fashion around the normal ducts. The tumor cells were small, with scant to moderate cytoplasm and round to ovoid nucleus. A few cells showed intracytoplasmic lumina. Mitoses were infrequent. Foci of Lobular Carcinoma In Situ (LCIS) were also seen. In addition to areas with typical morphology of invasive lobular carcinoma, areas of sclerosed fibroadenoma were also seen in areas adjoining the tumor (Figure 3). Eight out of 10 axillary lymph nodes on right side, and 7 out of 11 on the left side showed metastasis and measured 4 to $16 \mathrm{~mm}$. Immunohistochemically, the cells were estrogen receptor(ER) positive (70\% to $80 \%$ of the cells were positive with staining intensity $3+$ ), progesterone receptor(PR) positive $(60 \%$ to $70 \%$ of the cells were positive with staining intensity $3+]$, human epidermal growth factor receptor 2 (HER 2) negative, and E-cadherin negative. The Ki-67 index was $<10 \%$. A final diagnosis of bilateral stage III $b$ (T4 N2 Mo) invasive lobular carcinoma arising within a fibroadenoma was made. Following surgery, the patient received completion adjuvant chemotherapy (3 cycles of CAF regimen). Radiotherapy was started 4 weeks after surgery; 50 Gy was administered in 5 weeks ( 5 fractions/week, 2Gy per fraction) followed by $20 \mathrm{~Gy}$ boost to tumor bed. As the tumor was positive for estrogen and progesterone receptors, tamoxifen $20 \mathrm{mg}$ OD was also started. The patient was free from local recurrence and distant metastasis on imaging studies at 48 months of follow up.

Informed consent: written informed consent was obtained from the patient.

\section{Discussion}

Fibroadenoma usually occurs in young females under the age of 30. Complex lesions are more common in the older age group, with a peak age of 42 to 44 years. A diagnosis of fibroadenoma based on clinical assessment alone is insufficient. It is inaccurate in $27 \%$ to $50 \%$ of the cases (3). Patients with fibroadenoma have 1.3- to 2.1-fold higher risk of developing breast cancer than females in general population. Risk is further increased to 3.1- to 3.7-fold if fibroadenoma is complex in nature (4). Of all the carcinomas of the breast arising in a fibroadenoma, lobular carcinoma in situ is most common (50\%), followed by ductal carcinoma in situ (20\%), invasive ductal carcinoma (20\%), and invasive lobular carcinoma (10\%) (4). The patient had bilateral multiple simple fibroadenomas and malignant transformation occurred in invasive lobular carcinoma. Cheatle and Cutler in 1931 described a carcinoma arising in fibroadenoma for the first time. Azzopardi defined carcinomas involving fibroadenoma as "arising in the adjacent breast tissue engulfing and infiltrating fibroadenoma; in the crevices of a fibroadenoma as well as in the adjacent breast tissue; and carcinoma restricted entirely or at least dominantly to a fibroadenoma". In the literature, the mean age at diagnosis of breast carcinoma within fibroadenoma was 46.9 years (range, 27 to 80 years) and means tumor size was $2.46 \mathrm{~cm}$ (range, 0.8 to $5.1 \mathrm{~cm}$ ) (5). In the current case, the patient was much younger, with bilateral invasive lobular carcinoma, arising within fibroadenoma, and the lumps were significantly larger in size. This study found only one similar case in the literature, where a 43-yearold patient presented invasive lobular carcinoma with fibroadenoma on the left side, and invasive lobular carcinoma on the right side (6). This case report intends to highlight the risk of breast carcinoma in a fibroadenoma. However, the exact incidence of neoplastic transformation is difficult to determine as large tumors may eventually replace areas of fibroadenoma; the reported cumulative risk of carcinoma arising in a fibroadenoma is $0.7 \%$ after 5 years and $2.2 \%$ after 12 years, following a diagnosis of fibroadenoma (6). The malignant transformation of fibroadenoma is usually an incidental finding following the surgical removal of fibroadenoma or during FNAC of fibroadenoma. Carcinomas arising within fibroadenomas may be easily missed on FNAC as the lesional area may remain untouched. Imaging techniques are also not helpful as radiological signs are usually not evident until a breach occurs in false capsule. In this case, malignancy was probably missed during FNAC, or it was not present at that time. Hence, all patients should be informed about this risk and a close clinical follow up is necessary. The safest clinical approach is to perform a tissue diagnosis of dominant solid mass. Excisional biopsy remains the gold standard in these cases. In patients with family history of breast or ovarian lesions, this risk is significantly higher and histological assessment is a must. The tumor size and histopathological characteristics should determine subsequent local therapy and the need for adjuvant therapy.

\subsection{Learning Points}

- Malignant change could occur within fibroadenoma; hence all patients should be informed about this risk. A close clinical follow up must be done, especially in complex or multiple fibroadenomas, and excisional biopsy is the treatment of choice. 

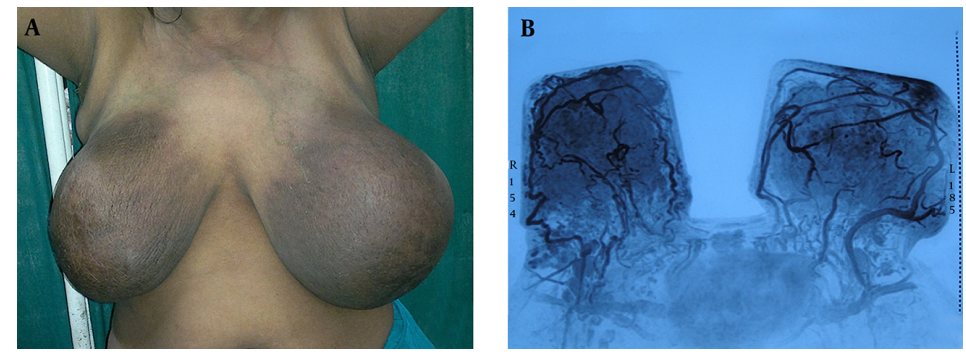

Figure 1. A, Lump Measuring Approximately $15 \times 15 \mathrm{~cm}$ in the Right Breast and $15 \times 13 \mathrm{~cm}$ in Left Breast With Skin Discoloration; B, Magnetic Resonance Imaging Breast With Angiography Revealed Bilateral Multinodular Masses in all Quadrants With Increased Angiogenesis.

Figure 2. A, Right Modified Radical Mastectomy Specimen Measuring $15 \times 14.5 \times 10.5 \mathrm{~cm}$; B, Left Modified Radical Mastectomy Specimen Measuring $15 \times 14 \times 5 \mathrm{~cm}$
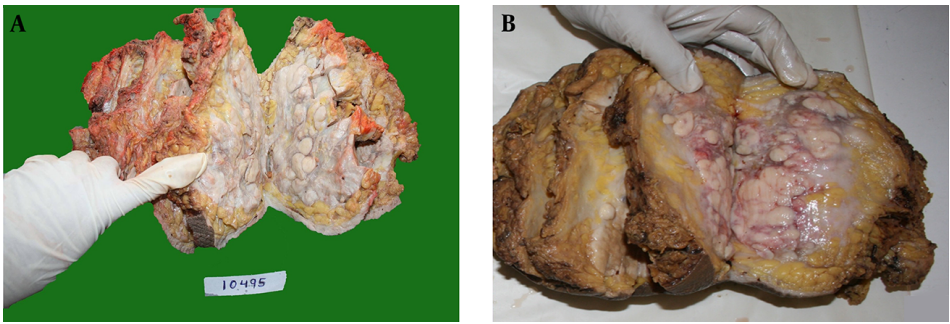

Bilateral tumor involved all four quadrants of the breast, hard in consistency, tan white in colour with foci of haemorrhage and necrosis
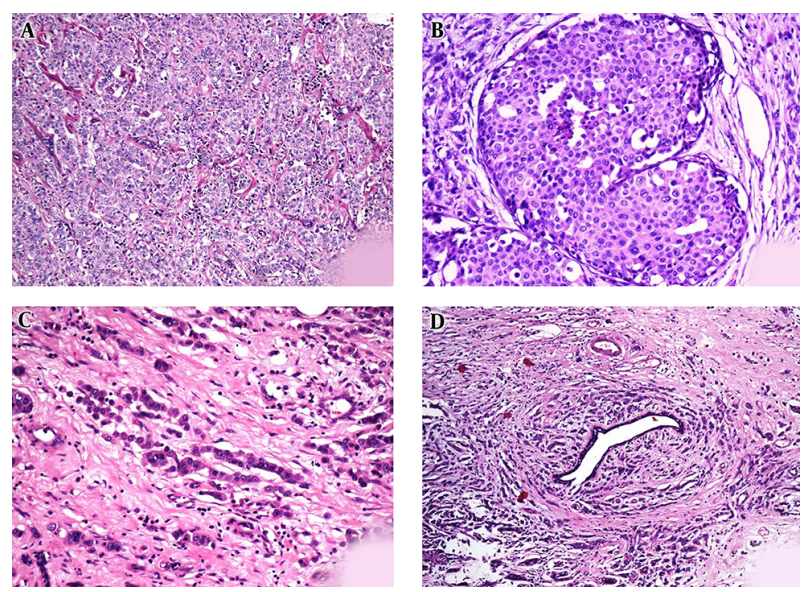

Figure 3. Hematoxylin and Eosin Stained Sections from Bilateral Breast Lesions Showed A, Invasive Lobular Carcinoma Disposed in Sheets with Cells Showing Minimal Pleomorphism; B, Lobular Carcinoma In Situ; C, Indian File Pattern; D, Tumor Cells Arranged in Concentric Fashion Around Normal Ducts (Targetoid Pattern).

- The biological behavior of carcinoma occurring within fibroadenoma does not differ from that of breast carcinoma unrelated to fibroadenoma.

- These cases can be managed aggressively with multimodality treatment similar to those with breast cancer.

\section{Acknowledgments}

The authors would like to thank Saanvi Bansal for her help. 


\section{Footnotes}

Conflict of Interests: The authors declared no conflict of interest.

Financial Disclosure: The authors declared that this study has received no financial support.

\section{References}

1. Goldhirsch A, Wood WC, Coates AS, Gelber RD, Thurlimann B, Senn HJ, et al. Strategies for subtypes-dealing with the diversity of breast cancer: highlights of the St. Gallen International Expert Consensus on the Primary Therapy of Early Breast Cancer 2011. Ann Oncol. 2011;22(8):173647. doi:10.1093/annonc/mdr304. [PubMed: 21709140].
2. Pick PW, Iossifides IA. Occurrence of breast carcinoma within a fibroadenoma. A review. Arch Pathol Lab Med. 1984;108(7):590-4. [PubMed: 6329129].

3. Wilkinson S, Anderson TJ, Rifkind E, Chetty U, Forrest AP. Fibroadenoma of the breast: a follow-up of conservative management. BrJ Surg. 1989;76(4):390-1. [PubMed: 2720350].

4. Sedloev T, Korukov B, Terziev I, Damyanov D. Fibroadenoma within lobular carcinoma in a woman with bilateral invasive breast cancer: a case report and review of the literature. Breast Case. 2013;2:16-20.

5. Wu YT, Chen ST, Chen CJ, Kuo YL, Tseng LM, Chen DR, et al. Breast cancer arising within fibroadenoma: collective analysis of case reports in the literature and hints on treatment policy. World J Surg Oncol. 2014;12:335. doi: 10.1186/1477-7819-12-335. [PubMed: 25382741].

6. Levi F, Randimbison L, Te VC, La Vecchia C. Incidence of breast cancer in women with fibroadenoma. Int J Cancer. 1994;57(5):681-3. [PubMed: 8194875]. 